\title{
Effect of EGF and FGF on the expansion properties of human umbilical cord mesenchymal cells
}

\begin{abstract}
Mesenchymal stem cells have been increasingly introduced to have great potential in regenerative medicine, immunotherapy, and gene therapy due to their unique properties of self-renewal and differentiation into multiple cell lineages. Studies have shown that these properties may be limited and changed by senescence-associated growth arrest under different culture conditions. This study aimed to present the ability of some growth factors on human umbilical cord mesenchymal (hUCM) cells expansion and telomerase activity. To optimize hUCM cell growth, epidermal growth factor (EGF) and fibroblast growth factor (FGF) were utilized in culture media, and the ability of these growth factors on the expression of the telomerase reverse transcriptase (TERT) gene and cell cycle phases was investigated. TERT mRNA expression increased in the hUCM cells treated by EGF and FGF. So, the untreated hUCM cells expressed 30.49冊政.15\% of TERT, while EGF-treated cells expressed 51.82 田配 $2.96 \%$ and FGF-treated cells expressed 33.77 田政1.55\% of TERT. Exposure of hUCM cells to EGF or FGF also promoted the progression of cells from G1 to S phase of the cell cycle and induced them to decrease the number of cells entering the G2/M phase. Our study showed that EGF and, to a lesser extent, FGF amplify the proliferation and expansion of hUCM cells.
\end{abstract}

Keyword: Cell cycle; Growth factors; TERT; Umbilical cord matrix-derived cells 